\title{
POINTS DE CONTINUITÉ D'UNE FONCTION SÉPARÉMENT CONTINUE
}

\author{
GABRIEL DEBS
}

\begin{abstract}
Let $f: X \times Y \rightarrow \mathbf{R}$ be a separately continuous function defined on the product of a Baire space $X$ and a Hausdorff compact space $Y$. We prove that $f$ is jointly continuous at any point of $G \times Y$, for a dense $G_{\delta}$ subset $G$ of $X$, under one of the following assumptions: (i) If the Banach space $C(Y)$ is weakly $K$-analytic; or (ii) if $X$ contains a dense subset which is $K$-analytic.
\end{abstract}

Etant donnés deux espaces topologiques $X$ et $Y$ on notera par $\mathcal{N}(X, Y)$ la propriété suivant:

"Pour toute fonction $f: X \times Y \rightarrow \mathbf{R}$ séparément continue il existe un $G_{\delta}$ dense $G$ de $X$ tel que $f$ soit continue en tout point de $G \times Y$."

Cette propriété s'introduit naturellement à partir du Théorème de Namioka [7]:

Dans ce travail on s'intéresse aux espaces topologiques $X$ (resp. $Y$ ) vérifiant $\mathcal{N}(X, Y)$ pour tout $Y$ (resp. $X)$ appartenant à une classe (large) d'espaces topologiques.

1. Définitions. (a) On dira qu'un espace topologique $X$ vérifie $\mathcal{N}$ (ou que $X$ est de Namioka) s'il vérifie $\mathcal{N}(X, Y)$ pour tout $Y$ compact.

(b) On dira qu'un espace topologique $Y$ vérifie $\mathcal{N}^{*}$ (ou que $Y$ est co-Namioka) s'il vérifie $\mathcal{N}(X, Y)$ pour tout $X$ de Baire.

2. Remarques. Si $X$ vérifie $\mathcal{N}$ alors:

(a) (J. P. R. Christensen [4]) Pour toute fonction $f: X \times Y \rightarrow M$ séparément continu avec $Y$ compact et $M$ métrisable, il existe un $G_{\delta}$ dense $G$ de $X$ tel que $f$ soit continue en tout point de $G \times Y$.

(b) (J. Saint-Raymond [8]) $X$ est un espace de Baire dès qu'il est complètement régulier.

3. Exemples. Les exemples principaux déjà connus sont les suivants:

(a) (J. Saint-Raymond) Si $X$ est de Baire métrisable alors $X \in \mathcal{N}$.

(b) (J. Saint-Raymond) Si $X$ est de Baire séparable alors $X \in \mathcal{N}$.

(c) (M. Talagrand) Si $X$ est de Baire contenant un $K_{\sigma}$ dense alors $X \in \mathcal{N}$.

(d) (J. Calbrix et J. P. Troallic) Si $Y$ est à base dénombrable alors $Y \in \mathcal{N}^{*}$.

(e) (R. Deville) Si $Y$ est un compact d'Eberlein alors $Y \in \mathcal{N}^{*}$.

Received by the editors July 12, 1984 and, in revised form, December 12, 1984.

1980 Mathematics Subject Classification. Primary 54C05, 54E52, 54H05, 90D05; Secondary $46 \mathrm{E} 15$.

Key words and phrases. Separate and joint continuity, Baire spaces, $K$-analytic sets, topological games. 
Les résultats que nous établirons nous permettront d'ajouter les deux cas suivants qui généralisent $(\mathrm{c})$ et $(\mathrm{e})$ respectivement:

(f) $\mathrm{Si} X$ est de Baire et contient un $\mathcal{K}$-analytique dense alors $X \in \mathcal{N}$.

(g) Si $Y$ est un espace compact tel que $C(Y)$ soit $K$-analytique pour la topologie de la convergence simple, alors $Y \in \mathcal{N}^{*}$.

4. Terminologie et notations. Dans toute la suite on note par

$\Sigma$ : l'ensemble $\mathbf{N}^{\mathbf{N}}$ des suites infinis d'entiers.

$S$ : l'ensemble $\mathbf{N}^{(\mathbf{N})}$ des suites finies d'entiers.

$\Delta$ : l'ensemble $\{0,1\}^{\mathbf{N}}$ des suites infinies de 0 et 1 .

$D$ : l'ensemble $\{0,1\}^{(\mathbf{N})}$ des suites finies de 0 et 1 .

Sur $\Sigma \cup S$ on note par $\prec$ la relation d'extension des suites. Pour $s$ et $t \in S$ on note par $s, t$ la suite obtenue par concaténation de $s$ et $t$; si $t$ est réduit à un élément $k \in \mathbf{N}$ on notera $s, k$. La longueur de la suite $s \in S$ est notée $|s|$ et la restriction de $\sigma \in \Sigma$ au segment $[0, n]$ est notée $\left.\sigma\right|_{n}$. Enfin pour tout $s \in S$ on pose $\Sigma(s)=\{\sigma \in \Sigma: s \prec \sigma\}$.

Si $T$ et $X$ sont des espaces topologiques, on rappelle qu'une application $\Phi: T \rightarrow$ $\mathcal{K}(X)$ est dite s.c.s. si l'ensemble $\{t \in T: \Phi(t) \subset V=\varnothing\}$ est ouvert pour tout ouvert $V$ de $X$. Un espace topologique $X$ est dit $K$-analytique s'il existe une application $K: \Sigma \rightarrow \mathcal{K}(X)$ de $\Sigma$ dans $\mathcal{K}(X)$ (ensemble des compacts de $X$ ) qui est s.c.s. et telle que $X=\bigcup_{\sigma \in \Sigma} K_{\sigma}$. L'application $\sigma \mapsto K_{\sigma}$ est alors dite une représentation analytique de $X$.

Enfin $C_{s}(X)$ (resp. $C_{u}(X)$ ) désigne l'espace des fonctions continues sur $X$ muni de la topologie de la convergence simple (resp. uniforme si $Y$ est compact). Pour $\varphi \in \mathcal{C}(X)$ et $A \subset X$ on note $\|\varphi\|_{A}=\sup _{x \in A}|\varphi(x)|$.

5. Définition. On dira qu'une partie $A$ d'un espace topologique $X$ possède universellement la propriété de Baire (u.p.B.) dans $X$ si pour tout compact $K$ et tout $f: K \rightarrow X$ continue, $f^{-1}(A)$ possède la propriété de Baire (p.B.) dans $K$.

6. Remarques. (a) Si $A$ possède u.p.B. dans $X$ alors pour tout compact $K$ de $X$ la partie $A \cap K=i^{-1}(A)$ (si $i: K \rightarrow X$ est l'injection canonique) possède p.B. En particulier si $X$ est compact, $A$ possède la propriété de Baire forte.

(b) Si $A$ possède u.p.B. dans $X=X_{1} \times X_{2}$ (espace topologique produit) alors pour tout $x_{1} \in X_{1}$ la coupe $A\left(x_{1}\right)=\left\{x_{2} \in X_{2}:\left(x_{1}, x_{2}\right) \in A\right\}$ possède p.B. puisque $A\left(x_{1}\right)=f^{-1}(A)$ avec $f: x_{2} \mapsto\left(x_{1}, x_{2}\right)$. Ceci est faux en général si

(c) Enfin la propriété qui nous intéressera dans la suite est que la classe des parties possèdant u.p.B. dans $X$ forme une tribu stable par schémas de Souslin (qui contient évidemment les fermés); en particulier cette classe contient les parties K-analytiques et leurs complémentaires.

7. THÉORÈME. Soient $X$ un espace de Baire complètement régulier, $Y$ un espace compact et $f: X \times Y \rightarrow[0,1]$ séparément continue. On suppose qu'il existe une partie $B$ vérifiant:

(i) $\{f(x, \cdot) ; x \in X\} \subset B \subset C(Y)$.

(ii) $B$ possède (u.p.B.) dans $[0,1]^{Y}$ (muni de la topologie produit).

Alors il existe un $G_{\delta}$ dense $G$ de $X$ tel que $f$ soit continue en tout point de $G \times Y$.

DÉmonstration. Notons par $\tilde{X}$ le compactifié de Stone-Čech de $X$ et par $\tilde{f}: \tilde{X} \rightarrow[0,1]^{Y}$ le prolongement continue de l'application continue de $X$ dans $[0,1]^{Y}$ 
définie par $x \rightarrow f(x, \cdot)$. On pose $f(x, y)=(\tilde{f}(x))(y)$; c'est une application continue en $x$ pour tout $y \in Y$. Par hypothèse $X \subset A=\tilde{f}^{-1}(B)$ et $A$ possède la propriété de Baire dans $\tilde{X}$. Il existe donc $E$ un $G_{\delta}$ de $\tilde{X}$ tel que $E \subset A$ et que $A \backslash E$ soit maigre dans $\tilde{X}$, et par suite que $X \cap E$ soit un résiduel de $X$. Nous poserons $E=\bigcap_{n \in \mathbf{N}} E_{n}$ avec $E_{0}=\tilde{X}$ et $E_{n}$ ouvert dans $\tilde{X}$.

Pour tout $\varepsilon>0$, notons par $G_{\varepsilon}(f)$ la réunion dans $X$ des ouverts $U$ vérifiant:

$$
\forall x^{\prime}, x^{\prime \prime} \in U, \quad \forall y \in Y, \quad\left|f\left(x^{\prime}, y\right)-f\left(x^{\prime \prime}, y\right)\right| \leq \varepsilon .
$$

Il suffit de montrer que les $G_{\varepsilon}(f)$ sont denses dans $X$; en effet on vérifie facilement que tout point de $\bigcap_{n>0}\left(G_{1 / n} \times Y\right)$ est un point de continuité de $f$.

Fixons alors $\varepsilon>0$; quitte à se restreindre à un ouvert de $X$ il suffit de montrer que $G_{\varepsilon}(f)$ est non vide. Supposons le contraire, alors pour tout ouvert non vide $O$ de $\tilde{X}$ on a (puisque $X$ est dense dans $\tilde{X}$ ):

$$
\exists x_{0}, x_{1} \in O \cap X, \quad \exists y \in Y: \quad\left|f\left(x_{0}, y\right)-f\left(x_{1}, y\right)\right|>\varepsilon .
$$

Nous allons alors construire pour tout $n \in \mathbf{N}$ et $d \in\{0,1\}^{n}$ des ouverts $O_{d}$ de $\tilde{X}$ et des points $y_{d}$ de $Y$ vérifiant:

(1) $\bar{O}_{d} \subset E_{n}$,

(2) $O_{d, 0} \cup O_{d, 1} \subset O_{d}$

(3) $\forall x_{i} \in O_{d, i},\left|\tilde{f}\left(x_{0}, y_{d}\right)-\tilde{f}\left(x_{1}, y_{d}\right)\right|>\varepsilon$.

Pour $n=0$ on pose $O_{\varnothing}=E_{0}=\tilde{X}$. Supposons alors construit pour $d \in\{0,1\}^{n}$ un ouvert $O_{d}$ vérifiant (1) et nous allons construire $O_{d, i}$ et $y_{d}$ vérifiant $(1),(2),(3)$. Par hypothèse, on a:

$$
\exists a_{0}, a_{1} \in O_{d} \cap X, \quad \exists y_{d} \in Y, \quad \exists \delta>0: \quad\left|f\left(a_{0}, y_{d}\right)-f\left(a_{1}, y_{d}\right)\right|>\varepsilon+\delta .
$$

Comme l'ouvert non vide $V_{i}=\left\{x \in O_{d}:\left|\tilde{f}\left(x, y_{d}\right)-f\left(a_{i}, y_{d}\right)\right|<\delta / 2\right\}$ recontre dans $X$ le résiduel $X \cap E$, il suffit de prendre $O_{d, i}$ tel que $\varnothing \neq O_{d i} \subset \overline{O_{d, i}} \subset$ $V_{i} \cap E_{n+1}$, qui vérifient (1) à (3).

Considérons alors le compact $L=\bigcap_{n \in \mathbf{N}} \bigcup_{|d|=n} \overline{O_{d}} \subset E$ et soit $M$ l'adhérence dans $C_{s}(L)$ de $\left\{\tilde{f}\left(\cdot, y_{d}\right) ; d \in D\right\}$. On conclut alors comme dans [10, Théorème 4.1]: $M$ est métrisable puisque c'est un compact séparable de fonctions continues sur un compact; et d'autre part il résulte de (3) que si $x_{i} \in O_{d, i}$ on a $\left\|\tilde{f}\left(x_{0}\right)-\tilde{f}\left(x_{1}\right)\right\|_{M} \geq \varepsilon$ ce qui contredit la séparabilité de $C_{u}(M)$.

Quoiqu'elle nous semble artificielle, l'hypothèse de complète régularité de $X$ est fondamentale dans la preuve précédente. Nous allons voir qu'en se restreignant à une propriété de Baire encore plus forte (stable par image directe continue), on peut supprimer cette hypothèse: ce sera le cas quand on supposera la partie $B$ du théorème, $K$-analytique. Mais ce n'est pas là la justification du théorème suivant, qui est plus précis et nous permettra de donner une nouvelle classe d'espaces de Namioka défini par des jeux.

Notations. Dans toute la suite on se donne une application séparement continue $f: X \times Y \rightarrow \mathbf{R}$ définie sur le produit de deux espaces topologiques $X$ et $Y$, et pour tout $\varepsilon>0$ on note par $G_{\varepsilon}(f)$ la réunion des ouverts $U$ de $X$ vérifiant:

$$
\forall x^{\prime}, x^{\prime \prime} \in U, \quad \forall y \in Y, \quad\left|f\left(x^{\prime}, y\right)-f\left(x^{\prime \prime}, y\right)\right| \leq \varepsilon .
$$


8. ThÉORÈme. Soient $X$ un espace de Baire, $Y$ un espace compact et $f: X \times$ $Y \rightarrow[0,1]$ séparément continue. On suppose que pour $\varepsilon>0$, il existe une partie $B$ de $\mathrm{C}(Y)$ vérifiant:

(i) $\left\{x \in X, \exists \varphi \in B:\|f(x, \cdot)-\varphi\|_{Y}<\varepsilon / 3\right\}$ contient un $G_{\delta}$ dense de $X$.

(ii) $B$ est $K$-analytique dans $C_{s}(Y)$. Alors l'ouvert $G_{\varepsilon}(f)$ est dense dans $X$.

DÉmonstration. Considérons dans le compact $K=[0,1]^{Y}$, pour tout $s \in S$, les parties $K_{s}$ et $B(s)$ définies par: $K_{s}=\overline{\bigcup_{\sigma \in \Sigma(s)} K_{\sigma}}$ et $B(s)=\bigcup_{\tau \in \Sigma(s)} \bigcap_{t \prec \tau} K_{t}$; il est bien connu alors que $K_{s}=\overline{B(s)}$ et $B(\varnothing)=B$.

Notons par $\hat{f}: X \rightarrow C_{s}(Y)$ l'application définie par $\hat{f}(x)=f(x, \cdot)$. Comme précedement il suffit de démontrer, par l'absurde, que $G_{\varepsilon}(f)$ est non vide; suppons donc le contraire:

Nous allons construire pour $n \in \mathbf{N}$ et $s \in\{0,1\}^{n}$ des ouverts non vides $U_{s}$ de $X$, des points $y_{s}$ de $Y$ et des suites finies d'entiers $\hat{s} \in \mathrm{N}^{|s|}$, vérifiant:

(1) $\forall x_{i} \in U_{s, i},\left|f\left(x_{0}, y_{s}\right)-f\left(x_{1}, y_{s}\right)\right|>\varepsilon$.

(2) $U_{s, i} \subset U_{s}$.

(3) $s \prec t \Rightarrow \hat{s} \prec \hat{t}$.

(4) L'ensemble $N_{s}=\left\{x \in U_{s}: d(\hat{f}(x), B(\hat{s})) \geq \varepsilon / 3\right\}$ est maigre dans $X$. Ici $d$ désigne la distance définie par la norme uniforme de $C(Y)$.

Pour $n=0$ et $s=\varnothing$ on pose $U_{\varnothing}=X$ et $\hat{\varnothing}=\varnothing$ de sorte que $B(\hat{\varnothing})=B$ et $N_{\varnothing}$ est maigre d'après (i), ce qui vérifie $(3),(4)$.

Supposons construits $U_{s}, \hat{s}$ vérifiant $(3)$ et (4) alors:

$$
\exists a_{0}, a_{1} \in U_{s}, \quad \exists y_{s} \in Y, \quad \exists \delta>0: \quad\left|f\left(a_{0}, y_{s}\right)-f\left(a_{1}, y_{s}\right)\right|>\varepsilon+\delta,
$$

et on définit $V_{i}=\left\{x \in U_{s}:\left|f\left(x, y_{s}\right)-f\left(a_{i}, y_{s}\right)\right|<\delta / 2\right\}$ ouvert non vide de $X$. Comme $B(\hat{s})=\bigcup_{k \in \mathbf{N}} B(\hat{s}, k)$, on a:

$$
V_{i}=\left(V_{i} \cap N_{s}\right) \cup \bigcup_{k \in \mathbf{N}}\left\{x \in V_{i}: d(\hat{f}(x), B(\hat{s}, k))<\varepsilon / 3\right\}
$$

l'ouvert $V_{i}$ n'étant pas maigre il résulte de (4) qu'il existe $k_{i} \in \mathbf{N}$ tel que l'ensemble:

$$
P_{i}=\left\{x \in V_{i}: d\left(\hat{f}(x), B\left(\hat{s}, k_{i}\right)\right)<\varepsilon / 3\right\}
$$

soit non maigre dans $X$.

Comme $F=\{(\varphi, \psi) \in K \times K:|\psi(y)-\varphi(y)| \leq \varepsilon / 3, \forall y \in Y\}$ est $\mathcal{K}_{\sigma}$ dans $K \times K$, il s'en suit que $\pi_{2}\left[F \cap\left(B\left(\hat{s}, k_{i}\right) \times K\right)\right]=A_{i}$ est $\bar{K}$-analytique dans $K$ (en désignant par $\pi_{2}$ la deuxième projection de $\left.K \times K\right)$ et par suite $\hat{f}^{-1}\left(A_{i}\right)$ est $\mathcal{F}$-souslinien dans $X$. En particulier $P_{i}=\hat{f}^{-1}\left(A_{i}\right) \cap V_{i}$ possède la propriété de Baire et on peut trouver un ouvert $U_{s, i} \subset V_{i}$ tel que $U_{s, i} \backslash P_{i}$ et $P_{i} \backslash U_{s, i}$ soient maigres (donc $U_{s, i} \cap P_{i}$ est non maigre et $U_{s, i}$ non vide). Enfin en prenant $(\widehat{s, i})=\left(\hat{s}, k_{i}\right)$ il est facile de vérifier (1) à (4).

D'après (4) l'ensemble:

$$
C(s)=\left\{\varphi \in B(\hat{s}): \exists x \in U_{s}:\|\hat{f}(x)-\varphi\|<\varepsilon / 3\right\}
$$

est non vide. En posant $L_{s}=\overline{C(s)} \subset K_{\hat{s}}$, on a: $L_{s} \supset L_{t}$ si $s \prec t$ de sorte que $L=\bigcap_{n} \bigcup_{|s|=n} L_{s}$ est un compact contenu dans $B$. On conclut enfin comme dans le Théorème précédent en considérant le compact métrisable

$$
M=\overline{\left\{y_{s} ; s \in\{0,1\}^{n} \text { et } n \in \mathbf{N}\right\}} \text {. }
$$


En effet si $\varphi_{i} \in C(s, i)$ et $x_{i} \in U_{s, i}$ tel que $\left\|\hat{f}\left(x_{i}\right)-\varphi_{i}\right\|<\varepsilon / 3$ on a d'après (1): $\left|\varphi_{0}\left(y_{s}\right)-\varphi_{1}\left(y_{s}\right)\right| \geq \varepsilon / 3$ et par passage à la limite on a l'inégalité précédente pour $\varphi_{i} \in L_{s, i}$ et par suite $\left\|\varphi_{0}-\varphi_{1}\right\|_{M} \geq \varepsilon / 3$ ce qui contredit la séparabilité de $C_{u}(M)$.

9. Un jeu topologique. Nous notons dans la suite par $J_{a}(X)$ le jeu suivant: $X$ est un espace topologique et le jeu est à deux joueurs $\alpha$ et $\beta$. C'est le joueur $\beta$ qui commence la partie en choisissant un ouvert non vide $V_{0}$; le joueur $\alpha$ doit alors choisir un couple $\left(U_{0}, A_{0}\right)$ où $U_{0}$ est un ouvert non vide contenu dans $V_{0}$ et $A_{0}$ une partie $K$-analytique de $X$. Au coup $n$ le joueur $\beta$ choisit un ouvert non vide $V_{n}$ puis le joueur $\alpha$ choisit un ouvert non vide $U_{n}$ et un ensemble $\mathcal{K}$-analytique $A_{n}$ avec la règle suivante:

$$
\left(V_{n+1} \subset U_{n} \subset V_{n}\right) \text { et }\left(A_{n} \subset A_{n+1}\right) .
$$

Le joueur $\alpha$ gagne la partie si $\beta$ ne respecte pas les règles ou si $\left(\bigcap_{n \in \mathbf{N}} V_{n}\right) \cap$ $\left(\overline{\bigcup_{p \in \mathbf{N}} A_{p}}\right) \neq \varnothing$. L'espace $X$ est dit $\alpha$-favorable pour $J_{a}$ si le joueur $\alpha$ possède une stratégie gagnante dans ce jeu. (Comme toujours, la notion de stratégie est à prendre au sens le plus faible c'est-à-dire que le jeu est supposé à information parfaite); sinon $X$ est dit $\alpha$-défavorable. On définit les notions de $\beta$-favorable et $\beta$-défavorable de manière analogue.

Ce jeu est une extension naturelle du jeu $J_{p}(X)$ de Saint-Raymond qui est défini de manière analogue avec la restriction que les $A_{n}$ sont des points $\left\{a_{n}\right\}$. Signalons que l'idée d'utiliser les jeux topologiques pour l'étude de la propriété $\mathcal{N}$ est dûe à J. P. R. Christensen qui avait introduit une version plus forte (c'est-à-dire un jeu moins favorable pour le joueur $\alpha$ ) de $J_{p}(X)$. On peut également considérer le jeu $J_{c}(X)$ correspondant au cas où on impose aux $A_{n}$ d'être compacts. Enfin en imposant que $A_{n}=X$ (pour tout $n$ ) on retrouve le jeu de Choquet $J(X)$ et dans lequel les joueurs $\alpha$ et $\beta$ jouent alternativement les ouverts $U_{n}$ et $V_{n}$. Notons par $J_{1} \prec J_{2}$ si le jeu $J_{1}$ est plus favorable pour le joueur $\alpha$ que le jeu $J_{2}$ c'est-à-dire vérifiant pour tout $X$ :

$$
\left\{\begin{array}{l}
J_{2}(X) \alpha \text {-favorable } \Rightarrow J_{1}(X) \alpha \text {-favorable } \\
\text { et } \\
J_{2}(X) \beta \text {-défavorable } \Rightarrow J_{1}(X) \beta \text {-défavorable. }
\end{array}\right.
$$

Alors on $a$ :

$$
J \prec J_{a} \prec J_{c} \prec J_{p} \text {. }
$$

En particulier puisqu'un espace est de Baire si et seulement si il est $\beta$-défavorable pour $J$ (voir [8]) on, alors tout espace $\beta$-défavorable pour $J_{i}(i=p, c, a)$ est de Baire. Enfin il découle immédiatement de ce qui précède que si $X$ est un espace de Baire contenant une partie dense qui est dénombrable (resp. $K_{\sigma}$, resp. $\mathcal{K}$-analytique) alors $X$ est $\beta$-défavorable pour $J_{p}$ (resp. $J_{c}$, resp. $\left.J_{a}\right)$.

10. THÉORÈME. Tout espace $\beta$-défavorable pour $J_{a}$ est un espace de Namioka.

La démonstration utilise le lemme suivant qui est implicite dans $[8$, Théorème 5].

LEMME. Soit $Y$ un espace compact et $B \subset C(Y) \cap\left([0,1]^{Y}\right)$, et notons par:

$$
\Gamma(B)=\left\{P\left(\varphi_{1}, \ldots, \varphi_{k}\right) ; k \in \mathbf{N}^{*}, P \in C\left([0,1]^{k}\right), \varphi_{i} \in B\right\} .
$$


Alors

$$
C(Y) \cap \bar{B}^{s} \subset \overline{\Gamma(B)}^{u} .
$$

DÉmonstration. Soient $\varphi \in \bar{B}^{s}$ et $\varepsilon>0$ et considérons l'application continue $\Phi: Y \rightarrow[0,1]^{B}$ définie par $\Phi(y)=\left((\Psi(y))_{\Psi \in B}\right)$ on a: $\left(\Phi(y)=\Phi\left(y^{\prime}\right) \Rightarrow \varphi(y)=\right.$ $\left.\varphi\left(y^{\prime}\right)\right)$ donc il existe $Q \in C\left([0,1]^{B}\right)$ tel que $\varphi=Q \circ \Phi$. Par le théorème de Stone il existe $F=\left\{\varphi_{1}, \ldots, \varphi_{n}\right\} \subset B$ et $P \in C\left([0,1]^{B}\right)$ tel que $\|P-Q\|<\varepsilon$ et $P$ ne dépend que des $F$-coordonnées, alors $\varphi^{\prime}=P \circ \Phi \in \Gamma(B)$ et $\left\|\varphi-\varphi^{\prime}\right\|<\varepsilon$.

DÉMONSTRATION DU THÉORÈME 10 . Soit $X$ un espace $\beta$-défavorable pour $J_{a}$ et considérons $f: X \times Y \rightarrow[0,1]$ séparement continue et $\hat{f}$ l'application induite de $X$ dans $C_{s}(Y)$.

Supposons que pour un $\varepsilon>0$ l'ouvert $G_{\varepsilon}(f)$ ne soit pas dense dans $X$, il existe alors un ouvert non vide $V_{0}$ tel que $V_{0} \cap G_{\varepsilon}(f)=\varnothing$.

Considérons un couple $(U, A)$ ou $U$ est un sous-ouvert non vide de $V_{0}$ et $A$ un K-analytique de $X$, alors l'ensemble $\Gamma(\hat{f}(A))$ défini dans le lemme précédent est $K$-analytique dans $C_{s}(Y)$; en effet l'application

$$
\Gamma_{k}: C_{u}\left([0,1]^{k}\right) \times\left[C_{s}(Y,[0,1])\right]^{k} \rightarrow C_{s}(Y)
$$

définie par

$$
\Gamma_{k}\left(P, \varphi_{1}, \ldots, \varphi_{k}\right)=P\left(\varphi_{1}, \ldots, \varphi_{k}\right),
$$

est continue et par suite

$$
\Gamma(\hat{f}(A))=\bigcup_{k \in \mathbf{N}} \Gamma_{k}\left(C_{u}\left([0,1]^{k}\right) \times(\hat{f}(A))^{k}\right)
$$

est $K$-analytique.

Remarquons que l'ensemble

$$
\pi(U, A)=\left\{x \in U: \forall \varphi \in \Gamma(\hat{f}(A)),\|\hat{f}(x)-\varphi\|_{Y} \geq \varepsilon / 3\right\}
$$

est non maigre dans $X$, car sinon en appliquant le Théorème 8 avec " $X=U$ " et " $B=\Gamma(\hat{f}(A))$ " on aurait que $G_{\varepsilon}(f) \cap U \neq \varnothing$ ce qui est impossible d'après le choix de $V_{0}$.

D'autre part par le même argument que dans le Théorème 8 on voit que l'ensemble $C=\left\{\psi \in K: \exists \varphi \in \Gamma(\hat{f}(A)),\|\psi-\varphi\|_{Y}<\varepsilon / 3\right\}$ (en posant $K=[0,1]^{Y}$ muni de sa topologie produit) est $K$-analytique, et par suite $\pi(U, A)=U \cap \hat{f}^{-1}(K \backslash C)$ possède la propriété de Baire dans $X$, puisque $C$ est $K$-souslinien.

Il existe alors une suite décroissante $\gamma_{p}(U, A)$ d'ouverts de $U$ vérifiant:

(1) $\left[\gamma_{p}(U, A) \backslash \pi(U, A)\right] \cup\left[\pi(U, A) \backslash \gamma_{p}(U, A)\right]$ est maigre,

(2) $\bigcap_{p \in \mathbf{N}} \gamma_{p}(U, A) \subset \pi(U, A)$.

Nous allons maintenant définir une stratégie $t$ pour $\beta$ en posant:

$$
\left\{\begin{array}{l}
t(\varnothing)=V_{0} \\
t\left(\left(U_{p}, A_{p}\right)_{0 \leq p \leq n}\right)=\bigcap_{0 \leq p \leq n} \gamma_{n}\left(U_{p}, A_{p}\right)=V_{n+1} .
\end{array}\right.
$$

Le seul point à vérifier est que $V_{n+1} \neq \varnothing$. En remarquant que $\pi\left(U_{p}, A_{p}\right) \subset$ $\pi\left(U_{p-1}, A_{p-1}\right)$ pour tout $p \leq n$, on a:

$$
\begin{aligned}
\gamma_{n}\left(U_{n}, A_{n}\right) \backslash \gamma_{n}\left(U_{p}, A_{p}\right) & \subset\left[\gamma_{n}\left(U_{n}, A_{n}\right) \backslash \pi\left(U_{p}, A_{p}\right)\right] \cup\left[\pi\left(U_{p}, A_{p}\right) \backslash \gamma_{n}\left(U_{p}, A_{p}\right)\right] \\
& \subset\left[\gamma_{n}\left(U_{n}, A_{n}\right) \backslash \pi\left(U_{n}, A_{n}\right)\right] \cup\left[\pi\left(U_{p}, A_{p}\right) \backslash \gamma_{n}\left(U_{p}, A_{p}\right)\right]
\end{aligned}
$$


ce qui montre d'après (1) que $\bigcup_{0 \leq p \leq n}\left[\gamma_{n}\left(U_{n}, A_{n}\right) \backslash \gamma_{n}\left(U_{p}, A_{p}\right)\right]$ est maigre, et comme il découle des remarques du début que $X$ est un espace de Baire alors $V_{n+1} \neq \varnothing$.

Le jeu $J_{a}$ étant $\beta$-défavorable le joueur $\alpha$ peut contrer cette stratégie donc il existe une partie $\left(V_{n} ;\left(U_{n}, A_{n}\right)\right)_{n \in \mathbf{N}}$ compatible avec $t$ et un point $x_{0}$ tel que pour $A=\bigcup_{n \in \mathbf{N}} A_{n}$ on ait:

$$
x_{0} \in \bigcap_{n \in \mathbf{N}} V_{n}=\bigcap_{0 \leq p \leq n} \gamma_{n}\left(U_{p}, A_{p}\right) \subset \bigcap_{p \in \mathbf{N}} \pi\left(U_{p}, A_{p}\right) \text { et } x_{0} \in \bar{A}
$$

par suite pour $\varphi_{0}=\hat{f}\left(x_{0}\right)$ on a dans l'espace métrique $\mathcal{C}_{u}(Y), \operatorname{dist}\left(\varphi_{0}, \Gamma\left(\hat{f}\left(A_{p}\right)\right)\right) \geq$ $\varepsilon / 3$ pour tout $p$, donc $\operatorname{dist}\left(\varphi_{0}, \Gamma(\hat{f}(A))\right) \geq \varepsilon / 3$ et d'autre part $\varphi_{0} \in \overline{\hat{f}}(A)^{s}$ ce qui contredit le lemme précédent.

11. COROLlaire. Tout espace de Baire contenant une partie $\mathcal{K}$-analytique dense est un espace de Namioka.

J. Saint Raymond a montré que tout espace $\beta$-défavorable pour $J_{p}$ est de Namioka; M. Talagrand a étendu ce résultat pour le jeu $J_{c}$ et R. Deville a montré que tout espace $\alpha$-favorable pour $J_{a}$ est $\mathcal{N}$. Dans ces différentes situations la stratégie à introduire dans la démonstration est très simple et ne nécessite pas l'appel à la propriété de Baire qui est un ingrédient fondamental dans la démonstration du Théorème 10.

Nous signalons une propriété de détermination des jeux topologiques considérés précédemment. Rappelons qu'un jeu est dit déterminé si l'un des deux joueurs possède une stratégie gagnante.

12. Proposition. Si $X$ est un espace de Baire $K$-analytique régulier alors $X$ est $\alpha$-favorable pour $J_{p}$.

DÉMONSTRATION. Soit $\sigma \rightarrow K_{\sigma}$ une représentation $K$-analytique de $X$ et posons pour tout $s \in S, F(s)=\overline{\bigcup_{\sigma \in \Sigma(s)} K_{\sigma}}$ fermé de $X$ (on rappelle que $K_{\sigma}=$ $\bigcap_{s<\sigma} F(s)$ pour tout $\left.\sigma \in \Sigma\right)$. Nous allons définir une stratégie $\rho$ pour $\alpha$ dans $J_{p}(X)$ : Si au premier $\operatorname{coup} \beta$ joue l'ouvert $V_{1}$ alors comme $V_{1} \subset \bigcup_{k \in \mathbf{N}} F(k)$ et que $V_{1}$ est de Baire alors:

$$
\exists k_{1} \in \mathbf{N}: \quad \operatorname{Int}\left(V_{1} \cap F\left(k_{1}\right)\right) \neq \varnothing
$$

alors $\alpha$ joue $\left(U_{1}, x_{1}\right)$ avec

$$
x_{1} \in U_{1} \subset \bar{U}_{1} \subset\left(V_{1} \cap F\left(k_{1}\right)\right) .
$$

De proche en proche le joueur $\alpha$ forme des triplets $\left(U_{n}, x_{n}, s_{n}\right)$ avec

$$
\left\{\begin{array}{l}
x_{n} \in U_{n} \subset \bar{U}_{n} \subset\left(V_{n} \cap F\left(s_{n}\right)\right), \text { et } \\
s_{n} \succ s_{n-1}, \quad s_{n} \in S,
\end{array}\right.
$$

en notant par $V_{n}$ le jeu de $\beta$ au $n^{\text {iéme }}$ coup. Ceci définit clairement une stratégie gagnante pour $\alpha$ dans $J_{p}(X)$. En effet si $K_{\sigma}=\bigcap_{n \in \mathbf{N}} F\left(s_{n}\right)$ alors $L=K_{\sigma} \cup\left\{x_{n} ; n \in\right.$ $\mathbf{N}$ \} est compact (en effet tout voisinage de $K_{\sigma}$ contient les points $x_{n}$ sauf au plus un nombre fini d'entre eux), et si $x$ est une valeur d'adhérence quelconque de la suite $\left(x_{n}\right)_{n \in \mathbf{N}}$ alors $x \in \bigcap_{n \in \mathbf{N}} U_{n}$. 
13. Corollaire. Si $X$ est un espace $\mathcal{K}$-analytique régulier alors les jeux $J(X), J_{a}(X), J_{c}(X)$ et $J_{p}(X)$ sont déterminés.

14. Fonctions séparément semicontinues. Dans ce paragraphe on étudie une extension de la propriété $\mathcal{N}$ au cas où la fonction $f$ est supposée séparément semicontinue. En fait il est facile de construire sur $[0,1] \times[0,1]$ une fonction séparément s.c.s. et qui n'a aucun point de continuité; cependant nous démontrons ici que si $f$ est s.c.s. en une variable et s.c.i. en l'autre, alors on a une conclusion analogue à celle de $\mathcal{N}$.

On rappelle que si $X$ et $Z$ sont deux espaces topologiques, une application $\Phi: X \rightarrow \mathcal{K}(Z)$ de $X$ dans l'ensemble des parties compactes de $Z$ est dite s.c.i. (resp. s.c.s.) en un point $x_{0} \in X$ si pour tout ouvert $W$ de $Z$ tel que $\Phi\left(x_{0}\right) \cap W \neq \varnothing$ (resp. tel que $\Phi\left(x_{0}\right) \subset W$ ) l'ensemble $\{x \in X: \Phi(x) \cap W \neq \varnothing\}$ (resp. l'ensemble $\{x \in X: \Phi(x) \subset W\})$ est un voisinage de $x_{0}$. Une fonction $\varphi: X \rightarrow \mathbf{R}^{+}$est s.c.i. (resp. s.c.s.) en $x_{0}$ au sens classique si et seulement si $\Phi(x)=[0, \varphi(x)]$ définit une application de $X$ dans $\mathcal{K}\left(\mathbf{R}^{+}\right)$qui est s.c.i. (resp. s.c.s.) en $x_{0}$. Le lemme suivant est bien connu quand $Z$ est séparable:

15. Lemme. Soient $X$ un espace de Baire, $(Z, d)$ un espace métrique et $\Phi: X \rightarrow \mathcal{K}(Z)$ une application s.c.i. Alors $\Phi$ est s.c.s. en tout point d'un résiduel de $X$.

DÉmonstration. Pour toute partie $A$ de $Z$ et tout $\varepsilon>0$ notons $B(A, \varepsilon)=$ $\{z \in Z: \exists a \in A, d(a, z)<\varepsilon\}$ et soit $R_{\varepsilon}$ l'ensemble des points $x$ de $X$ tels que dans tout voisinage $U$ de $X$, il existe $x^{\prime} \in U$ tel que $\Phi\left(x^{\prime}\right) \not \subset B(\Phi(x), \varepsilon)$. Nous allons montrer, par l'absurde, que $R_{\varepsilon}$ est rare.

Supposons que $V_{0}=\operatorname{Int}\left(\bar{R}_{\varepsilon}\right)$ soit non vide, nous allons définir une stratégie $\tau$ pour $\beta$ dans le jeu $J(X)$, en posant $Y(\varnothing)=V_{0}$; et au coup $n$ où le joueur $\alpha$ joue l'ouvert $U_{n}$, le joueur $\beta$ construit d'abord un point $z_{n} \in Z$ puis l'ouvert $V_{n+1}$ de $X$ qui sera son jeu pour le coup $n+1$, tel que:

$$
\left\{\begin{array}{l}
\text { (1) } d\left(z_{n+1}, z_{i}\right) \geq 3 \varepsilon / 4, \quad \forall i \leq n, \\
(2) \quad V_{n+1}=\left\{x \in U_{n}: \Phi(x) \cap B\left(z_{n+1}, \varepsilon / 4\right) \neq \varnothing\right\} .
\end{array}\right.
$$

Il s'agit évidemment de montrer qu'un tel choix est possible. Supposons cette stratégie bien définie jusqu' au coup $n$ et soit $x^{\prime} \in U_{n} \cap R_{\varepsilon}$ (qui est non vide: puisque $\left.U_{n} \subset V_{0}\right)$. Alors il existe $x^{\prime \prime} \in U_{n}$ tel que $\Phi\left(x^{\prime \prime}\right) \not \subset B\left(\Phi\left(x^{\prime}\right), \varepsilon\right)$ on peut trouver alors $z_{n+1} \in \Phi\left(x^{\prime \prime}\right)$ avec $\operatorname{dist}\left(z_{n+1}, \Phi\left(x^{\prime}\right)\right) \geq \varepsilon$; donc pour tout $i \leq n$ on a (puisque $\left.x^{\prime} \in U_{n} \subset V_{i}\right) \Phi\left(x^{\prime}\right) \cap B\left(z_{i}, \varepsilon / 4\right) \neq \varnothing$ et par suite $d\left(z_{n+1}, z_{i}\right) \geq 3 \varepsilon / 4$; d'autre part l'ouvert $V_{n+1}$, défini par (2) est non vide puisqu'il contient $x^{\prime \prime}$, ce qui montre que $\tau$ est une stratégie pour $\beta$. Soit $\left(V_{n}\right)_{n \in \mathbf{N}}$ une partie compatible avec $\tau$ et qui soit gagnée part $\alpha$. Fixons $x \in \bigcap_{n \in \mathbf{N}} V_{n}$ et pour tout $n \in \mathbf{N}$ soit $z_{n}^{\prime} \in \Phi(x) \cap B\left(z_{n}, \varepsilon / 4\right)$ alors il découle de (1) que $d\left(z_{i}^{\prime}, z_{n}^{\prime}\right) \geq \varepsilon / 4$ pour tous $i<n$, ce qui est impossible puisque $\Phi(x)$ est compact.

Donc pour tout $\varepsilon>0$ on a que $R_{\varepsilon}$ est rare et en tout point

$$
x \in \bigcap_{n>0}\left(X \backslash R_{1 / n}\right)
$$

l'application $\Phi$ est s.c.s. puisque les ouverts $B(\Phi(x), 1 / n)$ forment un système fondamental de voisinage pour $\Phi(x)$. 
16. REMARQUE. Si on suppose de plus $Z$ séparable alors on a la même conclusion pour $X$ quelconque. En effet dans ce cas on peut se ramener facilement au cas où $\Phi$ est une fonction s.c.i. à valeurs réelles. D'ailleurs la démonstration précédente reste valable si on suppose $Z$ compact-cas auquel on peut se ramener si $Z$ est séparable - et la stratégie que nous avons défini est nécessairement perdante pour $\beta$ ( $X$ étant quelconque) par la précompacité de $(Z, d)$ ce qui permet de conclure.

17. THÉORÈmE. Soient $X$ un espace de Baire, $Y$ un espace à base dénombrable, et $(Z, d)$ un espace métrique. On suppose que $\Phi: X \times Y \rightarrow K(Z)$ vérifie les deux propriétés suivantes:

(i) $x \rightarrow \Phi\left(x, y_{0}\right)$ est s.c.i. sur $X$ pour tout $y_{0} \in Y$,

(ii) $y \rightarrow \Phi\left(x_{0}, y\right)$ est s.c.s. sur $Y$ pour tout $x_{0} \in X$.

Si de plus $Y$ ou $Z$ est compact alors il existe un $G_{\delta}$-dense $G$ de $X$ tel que $\Phi$ soit s.c.s. en tout point de $G \times Y$.

DÉMONSTRATION. (a) Supposons $Z$ compact: Fixons une base $\left(V_{n}\right)_{n \in \mathbf{N}}$ de la topologie de $Y$ et pour tout $n \in \mathbf{N}$ considérons $\Psi_{n}: X \rightarrow \mathcal{K}(Z)$ définie par $\Psi_{n(x)}=\overline{\bigcup_{y \in V_{n}} \Phi(x, y)}$. On vérifie immédiatement que les $\Psi_{n}$ sont s.c.i. sur $X$ donc par le lemme précédent il existe un résiduel $G$ de $X$ en tout point duquel tous les $\Psi_{n}$ sont s.c.s.

Soient $\left(x_{0}, y_{0}\right) \in G \times Y$ et $W$ un ouvert de $Z$ tel que $\Phi\left(x_{0}, y_{0}\right) \subset W$. Comme $y \rightarrow \Phi\left(x_{0}, y\right)$ est s.c.s. en $y_{0}$, on peut trouver un voisinage $V_{n}$ de $y_{0}$ et un ouvert $W^{\prime}$ de $Z$ tels que:

$$
\forall y \in V_{n}, \quad \Phi\left(x_{0}, y\right) \subset W^{\prime} \subset \overline{W^{\prime}} \subset W
$$

donc $\Psi_{n}\left(x_{0}\right) \subset W$ et par la s.c.s. de $\Psi_{n}$ en $x_{0}$ on peut trouver un voisinage $U$ de $x_{0}$ tel que $\Psi_{n}(x) \subset W$ pour tout $x \in U$, en particulier:

$$
\forall(x, y) \in U \times V_{n}, \quad \Phi(x, y) \subset W
$$

ce qui montre que $\Phi$ est s.c.s. en $\left(x_{0}, y_{0}\right)$.

(b) Supposons $Y$ compact: Il suffit de reprendre la démonstration précédente en remarquant que $\Psi_{n}(x)$ est compact comme sous fermé de $K=\bigcup_{y \in Y} \Phi(x, y)$ qui est compact.

18. Corollaire (J. Calbrix et J. P. Troallic). Tout espace à base dénombrable vérifie $\mathcal{N}^{*}$.

19. Corollaire. Soient $X_{1}$ et $X_{2}$ deux espaces de Baire à base dénombrable et $f: X_{1} \times X_{2} \rightarrow R$ vérifiant:

(i) $x_{1} \rightarrow f\left(x_{1}, x_{2}\right)$ est s.c.i. sur $X_{1}$ pour tout $x_{2} \in X_{2}$,

(ii) $x_{2} \rightarrow f\left(x_{1}, x_{2}\right)$ est s.c.s. sur $X_{2}$ pour tout $x_{1} \in X_{1}$.

Alors il existe deux $G_{\delta}$ denses $G_{1}$ et $G_{2}$ de $X_{1}$ et $X_{2}$ respectivement tels que $f$ soit continue en tout point de $G_{1} \times G_{2}$.

\section{REFERENCES}

1. J. Calbrix et J. P. Troallic, Applications séparément continues, C. R. Acad. Sci. Paris Sér. A 288 (1979), 647-648.

2. G. Choquet, Lectures on analysis, Vol. 1, Benjamin, New York and Amsterdam, 1969.

3. J. P. R. Christensen, Joint continuity of separately continuous functions, Proc. Amer. Math. Soc. 82 (1981), 455-461. 
4. _ Remarks on Namioka spaces and R. E. Johnson's theorem on the norm separability of the range of certain mappings (a paraitre).

5. _ Theorems of Namioka and Johnson type for u.s.c.o. set-valued mappings (a paraître).

6. R. Deville, Parties faiblement de Baire dans les espaces de Banach (a paraître).

7. I. Namioka, Separate and joint continuity, Pacific J. Math. 51 (1974), 515-531.

8. J. Saint-Raymond, Jeux topologiques et espaces de Namioka, Proc. Amer. Math. Soc. 87 (1983), 499-504.

9. C. Stegall, Gâteaux differentiation of functions on a certain class of Banach spaces, Functions Analysis: Surveys and Recent Results. III, Elsevier North-Holland, Amsterdam, 1984.

10. M. Talagrand, Deux généralisations d'un théorème de I. Namioka, Pacific J. Math. 81 (1979), 239-251.

11. __ Propriété de Baire et propriété de Namioka, Math. Ann. 270 (1985), 159-174.

ÉQuipe D'Analyse, Université Paris VI, 4, Place Jussieu, 75230 Paris-Cedex 05, FRANCE 